\title{
When endoscopy pulls chestnuts out of the fire: a rare case of Mirizzi's syndrome treated with laser lithotripsy by direct peroral cholangioscopy
}

Mirizzi's syndrome is a possible complication of chronic gallstone disease and surgery remains the preferred approach for treatment in symptomatic patients [1]. An endoscopic approach is usually considered only a "bridge-to-surgery" treatment for decompressing the common bile duct (CBD) by papillotomy and stent (or nasal bile drainage) placement [2]. Here we describe the potential role of holmium laser lithotripsy under direct peroral cholangioscopy in a singular case of Mirizzi's syndrome ( Video $\mathbf{1}$ ).

An 82-year-old man was admitted due to abdominal pain and jaundice. Biochemistry showed high transaminases and bilirubin levels. Computed tomography was suspicious for Mirizzi's syndrome with a large stone between the CBD and a badly recognizable gallbladder (\$Fig.1a). Considering the patient's severe comorbidity (chronic obstructive pulmonary disease and heart failure) and the high risks related to surgery and anesthesiology, according to the surgeon, we performed endoscopic retrograde cholangiopancreatography (ERCP).

ERCP showed Mirizzi's syndrome type III with cholecystobiliary fistula involving up to two-thirds of the CBD diameter with an impacted large stone ( $>$ Fig. 1b). Stone extraction using both the retrieval balloon and the Dormia basket failed. We decided to use the SpyGlass System (Boston Scientific, Massachusetts, United States) to fragment the impacted stone with holmium laser lithotripsy (Storz 25750220, 365- $\mu \mathrm{m}$-diameter fiber, $15 \mathrm{~Hz} / 20 \mathrm{~W})$. The green aiming beam allowed the stone to be targeted ( Fig.2a), with the laser fiber in contact with the stone until it was fragmented ( Fig.2b); all fragments were finally removed with the retrieval balloon ( Fig. 2c) being able to obtain complete CBD toilette, as confirmed at cholangioscopy and cholangiography ( $\mathbf{F i g . 2 d}$ ).
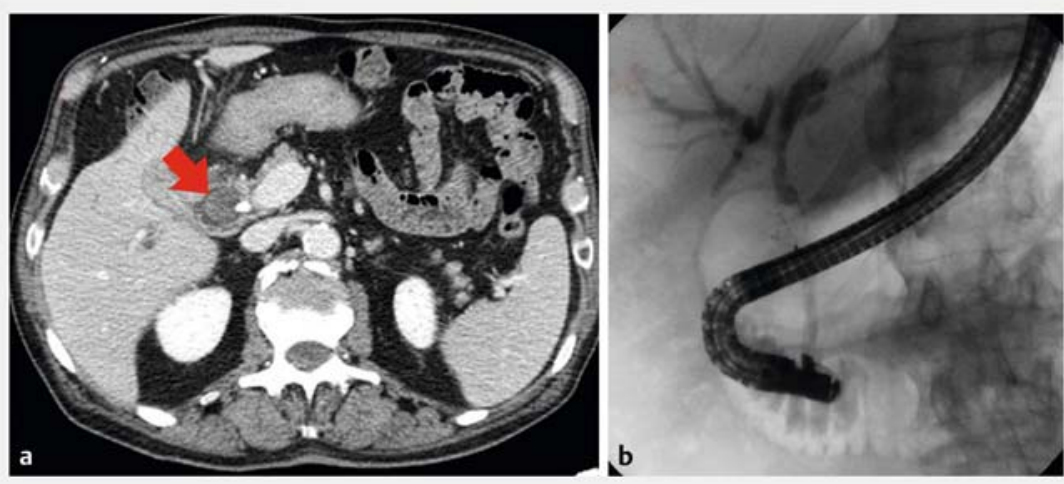

- Fig. 1 a CT scan of suspected a Mirizzi's syndrome with a large stone (red arrow) between the $\mathrm{CBD}$ and a badly recognizable gallbladder. b Cholangiography showing Mirizzi's syndrome type III with cholecystobiliary fistula involving up to two-thirds of the CBD diameter with an impacted large stone.
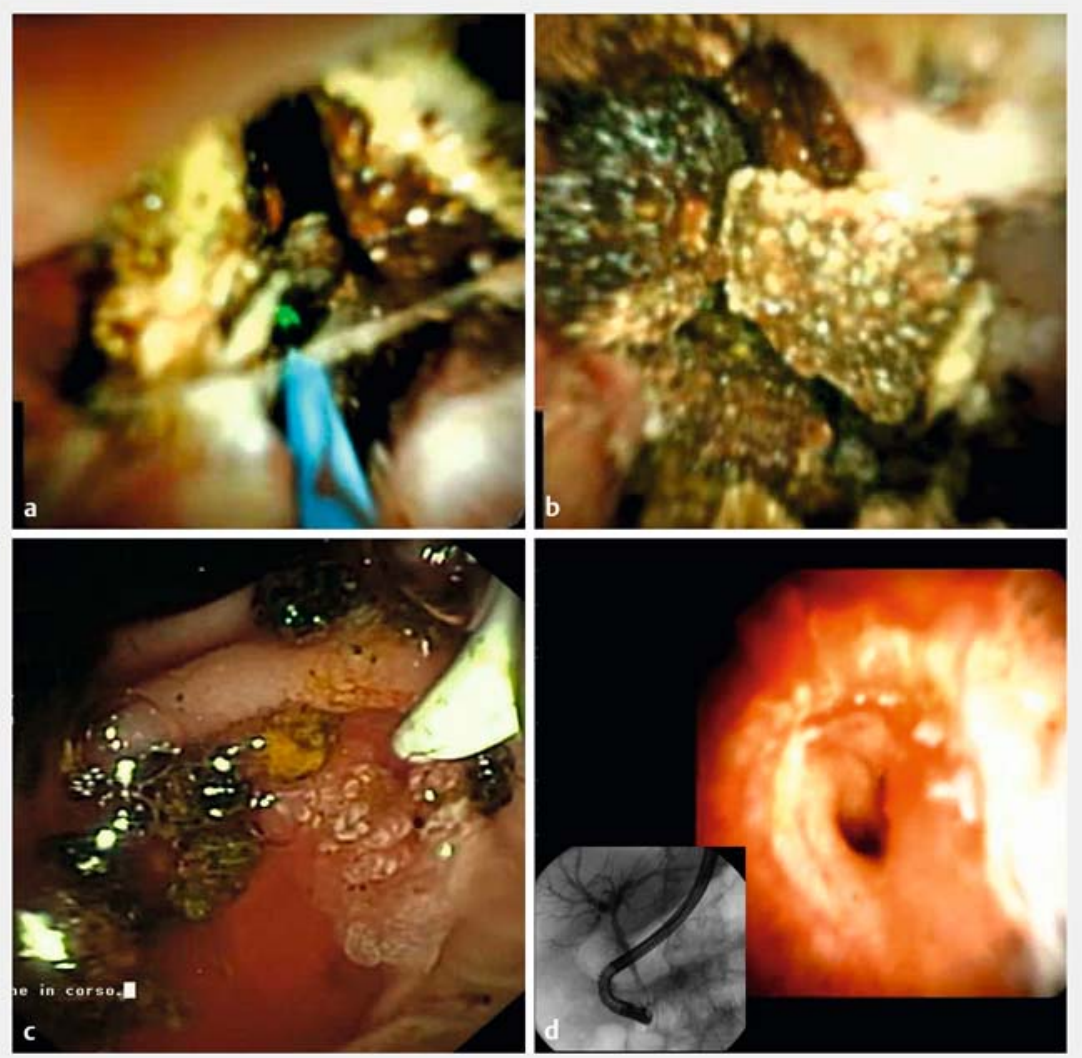

Fig. 2 a The green aiming beam of the laser targeting the stone. b Stone fragmentation. c Removed stone fragments. $\mathbf{d}$ complete CBD toilette at cholangioscopy and cholangiography. 


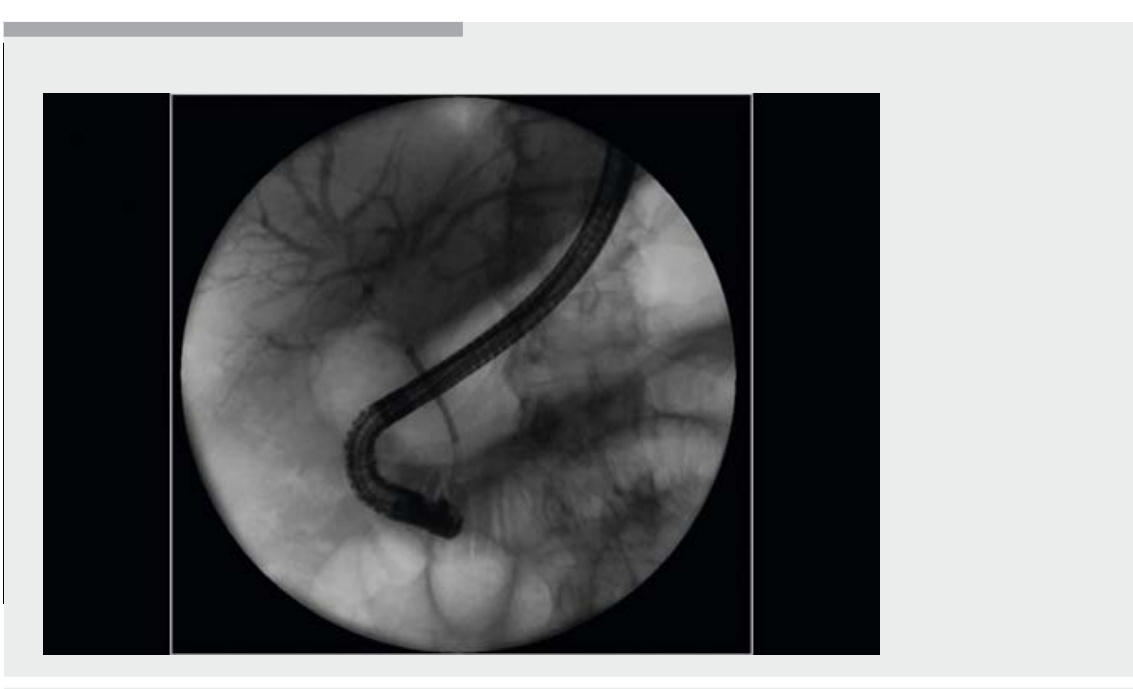

Video 1 Case of Mirizzi's syndrome treated with laser lithotripsy by direct peroral cholangioscopy.

No complications were reported and the patient was discharged after 3 days.

\section{Conclusion}

In conclusion, holmium laser lithotripsy through the SpyGlass System can help to remove impacted stones in the $\mathrm{CBD}$, allowing to avoid surgical treatment in selected patients with Mirizzi's syndrome.
The authors

Roberto Di Mitri, Filippo Mocciaro, Michela Lo Mastro, Ambra Bonaccorso, Elisabetta Conte, Daniela Scimeca

Gastroenterology and Endoscopy Unit, ARNAS Civico-Di Cristina-Benfratelli Hospital, Palermo, Italy

Corresponding author

\section{Filippo Mocciaro MD-PhD}

Via La Masa 29/E, 90011, Bagheria (PA), Italy Fax: +00390916663055

fmocciaro@gmail.com

\section{References}

[1] Alemi F, Seiser N, Ayloo S. Gallstone Disease: Cholecystitis, Mirizzi Syndrome, Bouveret Syndrome, Gallstone lleus. Surg Clin North Am 2019: 231-244

[2] Kumar A, Senthil G, Prakash A et al. Mirizzi's syndrome: lessons learnt from 169 patients at a single center. Korean J Hepatobiliary Pancreat Surg 2016; 20: 17-22

flict of interest.

\section{Bibliography}

DOI http://dx.doi.org/10.1055/a-1197-6725

Endoscopy International Open 2020; 08: E1115E1116

(C) Georg Thieme Verlag KG

Stuttgart · New York

eISSN 2196-9736 